\title{
Where Are We Going Wrong?
}

Exploring and Identifying the Challenges to Exclusive Breastfeeding in a Tertiary-Care Facility

Share this:

\author{
Sharanjit Kaur, $\mathrm{MPH}^{\mathrm{a}}$ \\ Julie Smith-Fehr, RN, BScN, MN ${ }^{b}$ \\ Jana Stockham, RN, BScN, IBCLC ${ }^{c}$ \\ Angela Bowen, RN, $\mathrm{PhD}^{\mathrm{d}}$
}

\begin{abstract}
Despite efforts to achieve the World Health Organization's best practice standards for a BabyFriendly status, only 54\% of babies discharged from a tertiary-care hospital in 2015 were exclusively breastfed. This is despite an initiation rate for breastfeeding of 92\%. This report describes maternal and nurse beliefs about exclusive breastfeeding, supplementation, and human milk banking. To help us understand our high levels of formula supplementation, we surveyed 94 mothers and 75 nurses and found differing beliefs about formula supplementation and the use of human donor milk. The breastfeeding policy was not being followed. Maternal and nurse breastfeeding education, along with up to date breastfeeding policies, are essential for organizations to meet the World Health Organization's standards to achieve Baby-Friendly status.
\end{abstract}

Keywords: breastfeeding; lactation; human milk; milk banks

The World Health Organization (WHO) recommends exclusive breastfeeding for all infants for the first 6 months of life (WHO, 2003); however, exclusive breastfeeding rates often lag behind this goal (Health Canada, 2012). In 1991, the WHO and United Nations Children's Fund (UNICEF) launched the Baby-Friendly Hospital Initiative (BFHI), which outlines the Ten Steps to Successful Breastfeeding to protect, promote, and support breastfeeding (WHO, 2013). These ten steps include sustained improvement in institutional practices, training healthcare staff to implement policies, educate pregnant women about the benefits of breastfeeding, practice rooming-in, including skin-to-skin contact after birth, and avoid unnecessary formula supplementation (WHO, 1998).

Exclusive breastfeeding promotes the health and wellbeing of both mother and infant. It protects the baby from illness and provides physical, cognitive, and social support to the baby and its family (Health Canada, 2015). Exclusive breastfeeding includes human milk (expressed or donor), oral rehydration solution (ORS), and syrups (i.e., vitamins, minerals, and medicines), but it does not include supplementation with other feeding products (WHO, 2015).

a.sharanjit.kaur@usask.ca

b. julie.smithfehr@saskatoonhealthregion.ca

c. jana.stockham@saskatoonhealthregion.ca

d. angela.bowen@usask.ca
Breastfeeding initiation rates vary internationally. In the United States, $81.1 \%$ of women initiate breastfeeding (Centers for Disease Control and Prevention, 2016), $70.1 \%$ in France (Organisation for Economic Cooperation and Development, 2009), 73.9\% in England (NHS England, 2014), 97\% in Norway (Australian Government Department of Health, 2012), and 96\% in Australia (Australian Institute of Health and Welfare, 2011). In Canada, the breastfeeding initiation rate is 89\% (Gionet, 2013).

In our facility, $92 \%$ of women initiate breastfeeding within an hour after birth, but by discharge, the breastfeeding rate falls to $88 \%$ (Saskatoon Health Region [SHR], 2015), and of this $88 \%$, only $54 \%$ of babies were exclusively breastfed, with $46 \%$ having been supplemented with formula (SHR, 2015). At the same time, infant formula use on the maternalinfant units increased from 13,022 bottles in 2013 to 21,254 in 2015 (SHR, 2015). The Human Milk Banking Association of North America (HMBANA) recommends that if the supply of maternal milk is insufficient, especially for the high-risk and premature infants, pasteurized human milk is the most appropriate supplementation. Milk banks play an important role in meeting needs of these babies (HMBANA, 2016). However, we do not have a formal supply of human donor milk for breast milk supplementation. As a result, in our community, human milk may be obtained from relatives, friends, or by human milk sharing on social media. 
Hospital administrators sought to understand the reasons for the gap between the goals of the promoted Breastfeeding policy and the infant feeding outcomes on the maternal-infant units in our region that includes a tertiary-care hospital and home visiting community program: specifically, the low rates of exclusive breastfeeding and high rates of supplementation. Therefore, we explored maternal and nurse knowledge and beliefs about exclusive breastfeeding, the reasons for supplementation, and the use of a human milk bank.

\section{Method}

This cross-sectional study involved mothers and nurses from maternal-infant units within a tertiary-care Canadian hospital and home visiting community program with approximately 5,500 live births in 2015 .

\section{Participants}

Participants included a convenience sample of nurses from our maternal-infant units and mothers from the in-patient postpartum ward maternal-infant units. Approval was received from the Saskatoon Health Region and University of Saskatchewan Behavioral Ethics Committees. Informed consent was received from all participants.

\section{Inclusion and Exclusion Criteria}

Women 18 years or older and who speak English, or had a family member available to translate the survey, were included. The goal of the study was to identify the challenges to exclusive breastfeeding. Therefore, we only included mothers whose babies had received formula supplementation during their in-patient stay. Exclusively breastfeeding mothers whose infants had not received supplementation were excluded from the study. All nurses who worked on the maternal-infant units were invited to participate with no exclusions.

\section{Data Collection}

Mothers were interviewed and filled out the questionnaires in their in-patient room prior to discharge home, over a 2-week period. Nurses were asked to complete the survey during their work shifts. Data were also gathered from the maternal and infant in-patient charts.

\section{Questionnaire}

The mother's interview included 22 questions including age, gravida, gestational age, and weight of baby at birth. We asked about challenges the mothers faced to exclusively breastfeed, the reasons for supplementation, and views about human milk as an alternative source of nutrition for their baby. The nurses' survey tested their breastfeeding knowledge via 20 multiple-choice questions. We also asked their beliefs on exclusive breastfeeding, formula supplementation, free formula supply to hospital, and the use of human milk banking.

\section{Analysis}

Descriptive statistics were used. The nurses' knowledge was divided into three categories: those below 50\%, those between $51 \%$ and $75 \%$, and those who scored up to $100 \%$. Data were analyzed using the latest version of SPSS Version 22.

\section{Results}

\section{Mothers}

Ninety-four mothers were interviewed; $67 \%(n=63)$ of women had vaginal deliveries. Fifty-seven percent $(n=$ 54 ) of the women were primigravidas, $40 \%$ of mothers $(n=38)$ were multigravidas, and $3 \%(n=2)$ of women did not answer this question (Table 1). Seventy-four percent $(n=70)$ of the mothers stated their babies received supplementation because of a medical condition. Eleven percent $(n=10)$ stated that supplementing their baby was personal choice, and $13 \%(n=12)$ of the women did not know why their babies had received formula. Fortyfive percent $(n=42)$ of the mothers stated inadequate milk supply was their biggest breastfeeding challenge, followed by breast pain $14 \%(n=14)$, sore nipples $11 \%$ $(n=11)$, and infant tongue-tie $11 \%(n=10)$.

Of the multiparous mothers, $63 \%(n=24)$ had also supplemented their first baby. Fifteen percent $(n=15)$ of mothers practiced skin-to-skin contact after delivery, whereas $40 \%(n=37)$ were separated from their babies, but this was for less than 20 minutes, $10 \%(n=9)$ of mothers did not have any skin-to-skin contact with their babies despite not being separated from them after birth. The in-patient charts showed that the main reason noted for supplementation was low blood sugar, at 36\% $(n=33)$, followed by admission to neonatal care $32 \%$ $(n=30)$. Factors such as maternal illness and infant dehydration were other reasons, contributing $4.2 \%$ $(n=4)$ and $6.3 \%(n=6)$, respectively.

Almost half $(n=48)$ of the mothers stated that pasteurized human milk from a milk bank would be the preferred method to supplement their baby. Half of mothers (50\%) were unsure about using human milk from a milk bank because of the unknown medical history of the donor mother and lack of knowledge regarding pasteurization of the collected milk. Two mothers reported using unpasteurized donor human 


\begin{tabular}{|c|c|}
\hline & $\%(n)$ \\
\hline Age (years) & \\
\hline $18-24$ & $26.60(25)$ \\
\hline $25-34$ & $50.00(47)$ \\
\hline $35-44$ & $20.21(19)$ \\
\hline 45 or older & $2.13(2)$ \\
\hline Type of delivery & \\
\hline Vaginal & $67.02(63)$ \\
\hline Caesarean & $31.91(30)$ \\
\hline Gestational age of baby & \\
\hline Less than 32 weeks & $2.13(2)$ \\
\hline Less than 37 weeks & $30.85(29)$ \\
\hline 37 weeks or after & $65.96(62)$ \\
\hline Weight of baby at the time of birth & \\
\hline$<1,500 \mathrm{~g}$ & $3.19(3)$ \\
\hline $1,500 \mathrm{~g}$ to $<2,500 \mathrm{~g}$ & $27.66(26)$ \\
\hline$>2,500 \mathrm{~g}$ & $67.02(63)$ \\
\hline Is this your first baby? & \\
\hline Yes & $57.40(54)$ \\
\hline No & $40.40(38)$ \\
\hline No answer & $3.00(2)$ \\
\hline $\begin{array}{l}\text { If this is not your first baby, was one of } \\
\text { your other babies supplemented? }\end{array}$ & \\
\hline Yes & $63.20(24)$ \\
\hline No & $36.80(14)$ \\
\hline $\begin{array}{l}\text { Why was your baby supplemented with } \\
\text { formula? }\end{array}$ & \\
\hline Medical reasons & $74.47(70)$ \\
\hline Personal choice & $10.64(10)$ \\
\hline Fussy baby & $8.51(8)$ \\
\hline I don't know & $12.77(12)$ \\
\hline
\end{tabular}

milk for their baby, and 3 were using home methods of pasteurized human milk. The remaining 84 denied accessing any human milk for supplementation.

\section{Nurses}

Seventy-five nurses completed the survey; $95 \%(n=72)$ answered more than $50 \%$ of the multiple-choice questions correctly, that is, $45 \%(n=34)$ scored up to $100 \%$ and
$50 \%(n=38)$ scored up to $75 \%$, only $5 \%(n=3)$ of the nurses received a mark less than $50 \%$. Fifty-two percent $(n=39)$ of the nurses reported that they do not have enough time to teach mothers about exclusive breastfeeding, $42 \%(n=32)$ said they sometimes have time, whereas only $5 \%$ agreed that they do get enough time to educate mothers about exclusive breastfeeding. Most nurses stated that infant formula use was mostly because of mother's choice $(62 \%, n=47)$, followed by medical reasons $(37 \%, n=28)$, inadequate education to mothers $(25 \%, n=19)$, and the availability of free infant formula $(6 \%, n=5)$.

Almost two-thirds $(n=47)$ of the nurses said that breastfeeding is better for babies than infant formula, whereas $29 \%(n=22)$ stated that formula is a very good alternative to breast milk. Eight percent of nurses $(n=6)$ think that maternal preference should be the only deciding factor for formula use. Last, $90 \%(n=70)$ of nurses stated that a human milk bank would be beneficial for both mothers and infants.

\section{Mother-Nurse Comparison}

There were notable differences between the mothers' and nurses' knowledge and beliefs; 62\% $(n=47)$ of nurses think that for the majority of babies, infant formula is given as a result of the mother's personal choice. Of the mothers interviewed, only $11 \%(n=10)$ stated it was their personal choice. Only $37 \%$ of the nurses said that infant formula use was because of a medical condition of the mother or baby; however, $74 \%(n=70)$ of the mothers interviewed believed the baby was supplemented because of medical condition. These examples show a discrepancy in beliefs. When asked about use of a human milk bank for supplementation, $90 \%$ of nurses said it would be a good strategy, whereas only $50 \%$ of the mothers interviewed responded positively about using a human milk bank.

\section{Discussion}

Our study supports the findings of DiGirolamo, Grummer-Strawn, and Fein (2003) who state that mothers have a perceived rather than actual inadequate milk supply. In addition, nurses' lack of knowledge about the reasons for supplementation as well as the free formula supplied to the hospital all contribute to the low rates of exclusive breastfeeding.

Lack of breastfeeding knowledge is an ongoing concern for new mothers. The perception of insufficient milk production was a major concern voiced by the mothers in our study. This affirms Gatti (2008) who reports that 
the perception of lack of milk production is a leading issue for women across Canada.

According to UNICEF, the introduction of infant formula early in a baby's life reduces the chances a mother will resume exclusive breastfeeding (UNICEF, 2015). A 2015 study found that when hospitals had a policy of not accepting free infant formula, in-hospital formula supplementation was reduced and exclusive breastfeeding rates increased (Tarrant et al., 2015). At the time of this study this tertiary-care hospital is accepting free formula. Therefore, it is essential for administrators to realize that following the International Code of Marketing of Breast-Milk Substitutes for supplementation discourages the increasing use of formula (WHO, 1981), which might be contributing to the current low rates of exclusive breastfeeding.

The literature suggests that immediate, uninterrupted skin-to-skin contact for a minimum of an hour is one of the most effective interventions to promote exclusive breastfeeding and to elevate infant blood sugar levels (Crenshaw, 2014). This supports our findings that 10\% of the babies in our study did not remain in skin-to-skin contact despite no obvious cause for being separated from their mother (i.e., for maternal or infant medical reasons), and that most babies who were supplemented was because of low blood sugar level.

Repeating information to mothers and nurses about exclusive breastfeeding, milk expression, and proper supplementation (including access to human milk) can increase exclusive breastfeeding rates to the WHO standards of $75 \%$ exclusive breastfeeding and make achieving Baby-Friendly status possible (Martens, 2000). Our study also suggests that when women do not fully understand when or how to supplement, it likely impacts the rates of exclusive breastfeeding. This appears to have been influenced by the nurses who stated that they do not have time to teach the mothers about exclusive breastfeeding. Prenatal classes are an ideal venue to teach women about the importance of breastfeeding, options for supplementation, and to influence their beliefs about exclusive breastfeeding (Centers for Disease Control and Prevention, 2013).

\section{Limitations}

The mothers may have been concerned that their answers may reflect poorly on their mothering abilities and answered accordingly, which may have contributed to social desirability bias. Second, the small sample size may affect the generalizability of results, although the sample was consistent with the usual population of the unit. Third, a few mothers were not fluent in English, so family members gave answers on their behalf. Some might have understood the questions differently than was intended or did not want family to know their actual responses. Last, data were not divided by area of work; the maternal-infant in-patient area is staffed mostly with registered nurses, whereas the home visiting community program has predominantly registered nurses also trained as IBCLCs, with different knowledge, roles, and skill set.

\section{Conclusion}

This study provides insights into the challenges faced in trying to achieve exclusive breastfeeding and subsequent Baby-Friendly status in a tertiary-care facility. This includes gaps in the knowledge and beliefs of mothers and nurses related to exclusive breastfeeding and supplementation. Mothers need to understand the importance of exclusive breastfeeding and the practices that promote it such as appropriate supplementation, skin-to-skin contact, and human milk banking. Nurses need appropriate orientation, continuing education, and annual updates on best practices in breastfeeding. And they need to be provided with the time to ensure that postpartum mothers have the information necessary to make informed decisions about exclusive breastfeeding and avoid misperceptions about formula supplementation and human milk options (Carroll, 2014).

Increasing maternal and nursing knowledge, supporting existing policy, access to human donor milk, and policy changes to ensure that free formula is not accepted are essential to improve exclusive breastfeeding rates in order to meet the WHO's standards to achieve Baby-Friendly status.

\section{References}

Australian Government Department of Health. (2012). An international comparison study into the implementation of the WHO code and other breastfeeding initiatives. Norway-the WHO code and breastfeeding: An international comparative overview. Retrieved from http://www.health.gov.au/internet/main/publishing.nsf/ content/int-comp-whocode-bf-init

Australian Institute of Health and Welfare. (2011). 2010 Australian National Infant Feeding Survey: Indicator results. Canberra, Australian Capital Territory Australia: Author. Retrieved from http://www.aihw.gov.au/publication-detail/?id=10737420927

Carroll, K. (2014). Body dirt or liquid gold? How the 'safety' of donated breastmilk is constructed for use in neonatal intensive care. Social Studies of Science, 44(3), 466-485. Retrieved from https://www.ncbi.nlm.nih.gov/pubmed/25051591

Centers for Disease Control and Prevention. (2013). Strategies to prevent obesity and other chronic diseases: The CDC guide to strategies to support breastfeeding mothers and babies. Atlanta, GA: U.S. 
Department of Health and Human Services. Retrieved from https://www.cdc.gov/breastfeeding/pdf/BF-Guide-508.PDF

Centers for Disease Control and Prevention. (2016). Breastfeeding report card: Progressing toward national breastfeeding goals. United States, 2016. Retrieved from https://www.cdc.gov/ breastfeeding/pdf/2016breastfeedingreportcard.pdf

Crenshaw, J. T. (2014). Healthy Birth Practice \#6: Keep mother and baby together-it's best for mother, baby, and breastfeeding. The Journal of Perinatal Education, 23(4), 211-217. Retrieved from http://dx.doi.org/10.1891/1058-1243.23.4.211

DiGirolamo, A. M., Grummer-Strawn, L. M., \& Fein, S. B. (2003). Do perceived attitudes of physicians and hospital staff affect breastfeeding decisions? Birth, 30(2), 94-100.

Gatti, L. (2008). Maternal perceptions of insufficient milk supply in breastfeeding. Journal of Nursing Scholarship, 40(4), 355-363. http://dx.doi.org/10.1111/j.1547-5069.2008.00234.x

Gionet, L. (2013). Health at a glance. Breastfeeding trends in Canada. Ottawa, Ontario, Canada: Statistics Canada. Retrieved from http://www.statcan.gc.ca/pub/82-624-x/2013001/article/11879. eng.htm

Health Canada. (2012). Duration of exclusive breastfeeding in Canada: Key statistics and graphics (2009-2010). Retrieved from http://www.hc-sc.gc.ca/fn-an/surveill/nutrition/commun/ prenatal/exclusive-exclusif-eng.php\#share

Health Canada. (2015). Nutrition for healthy term infants: Recommendations from birth to six months. Retrieved from http://www.hc-sc.gc.ca/fn-an/nutrition/infant-nourisson/ recom/index-eng.php

Human Milk Banking Association of North America. (2016). Why bank milk? Retrieved from https://www.hmbana.org/

Martens, P. J. (2000). Does breastfeeding education affect nursing staff beliefs, exclusive breastfeeding rates, and Baby-Friendly Hospital Initiative compliance? The experience of a small, rural
Canadian hospital. Journal of Human Lactation, 16(4), 309-318. http://dx.doi.org/10.1177/089033440001600407

NHS England. (2014). Statistical release: Breastfeeding initiation $\mathcal{E}$ breastfeeding prevalence 6-8 weeks. Revised Quarter 4 2013/14. Retrieved from https://www.england.nhs.uk/statistics/wp-content/ uploads/sites/2/2014/03/Breastfeeding-1314-Revised-Data.pdf

Organisation for Economic Co-operation and Development. (2009). CO1.5: Breastfeeding rates. Retrieved from https://www.oecd .org/els/family/43136964.pdf

Saskatoon Health Region. (2015). Baby-friendly initiative external assessment of CHS. Saskatoon, Saskatchewan. (Report no. 24A).

Tarrant, M., Lok, K., Fong, D., Lee, I., Sham, A., Lam, C., . . . Dodgson, J. (2015). Effect of a hospital policy of not accepting free infant formula on in-hospital formula supplementation rates and breast-feeding duration. Public Health Nutrition, 18(14), 2689-2699. http://dx.doi.org/10.1017/S1368980015000117

United Nations Children's Fund. (2015). Breastfeeding. Retrieved from http://www.unicef.org/nutrition/index 24824.html

World Health Organization. (1981). International code of marketing of breast-milk substitutes. Retrieved from http://www.who.int/ nutrition/publications/infantfeeding/9241541601/en/

World Health Organization. (1998). Evidence for the ten steps to successful breastfeeding. Geneva, Switzerland: Author. Retrieved from http://www.who.int/nutrition/publications/ evidence ten step eng.pdf

World Health Organization. (2003). Exclusive breastfeeding for optimal growth, development and health of infants. Retrieved from http:// www.who.int/elena/titles/exclusive breastfeeding/en/

World Health Organization. (2013). Baby-friendly Hospital Initiative. Retrieved from http://www.who.int/nutrition/topics/bfhi/en/

World Health Organization. (2015). The World Health Organization's infant feeding recommendation. Retrieved from http://www.who .int/nutrition/topics/infantfeeding recommendation/en/

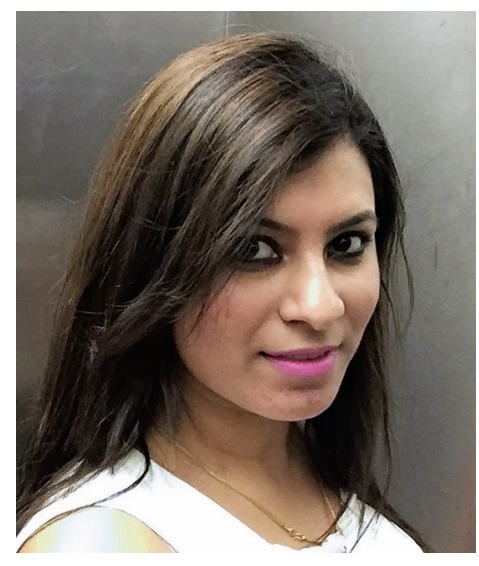

Sharanjit Kaur, MPH, is a public health professional with a master's degree from the University of Saskatchewan. She has a keen interest in addressing major health issues in aboriginal and vulnerable populations. 

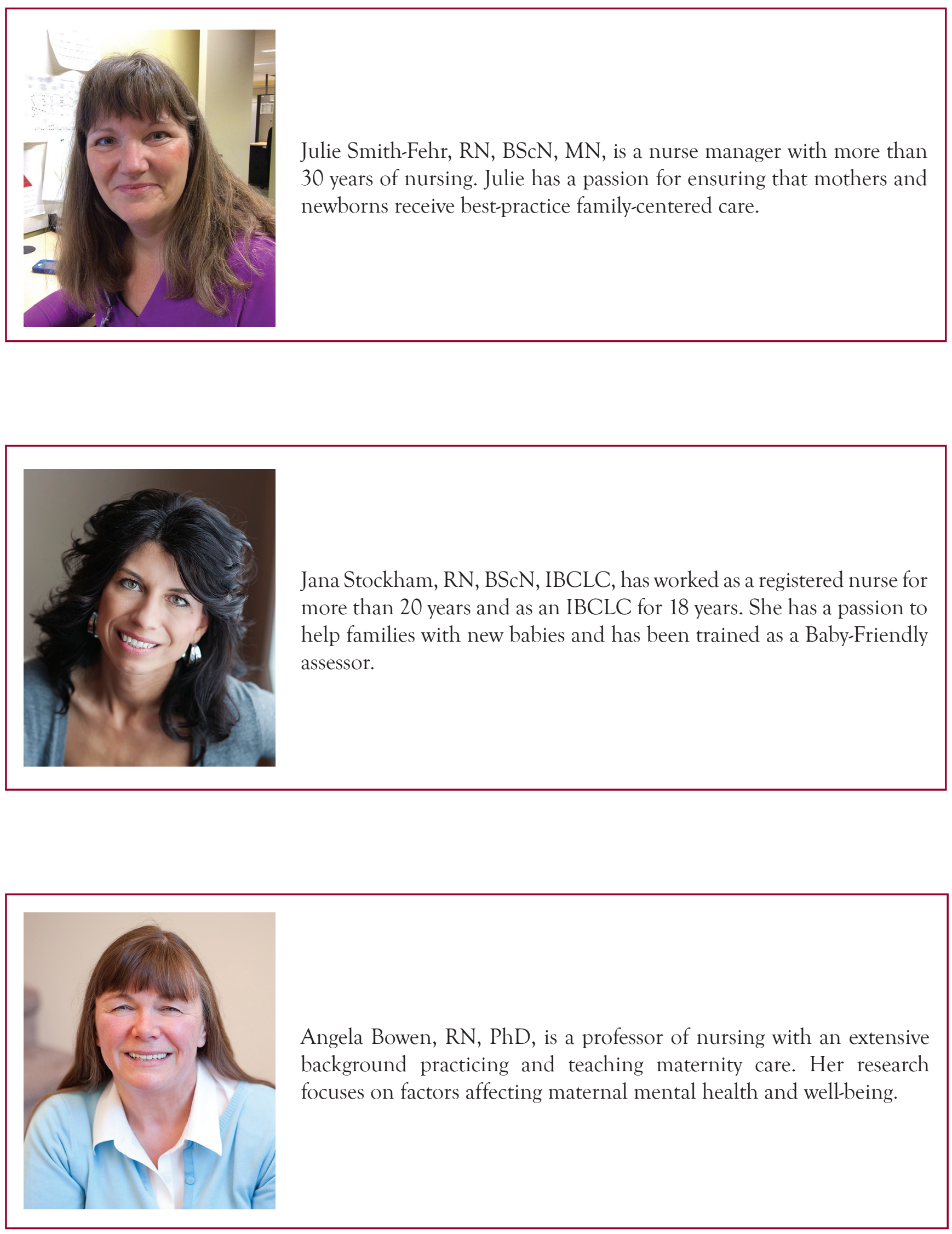